OPEN ACCESS

Check for updates

\title{
Association of first trimester prescription opioid use with congenital malformations in the offspring: population based cohort study
}

\author{
Brian T Bateman, ${ }^{1,2}$ Sonia Hernandez-Diaz, ${ }^{3}$ Loreen Straub, ${ }^{1}$ Yanmin Zhu, ${ }^{1}$ Kathryn J Gray, ${ }^{4}$ \\ Rishi J Desai, ${ }^{1}$ Helen Mogun, ${ }^{1}$ Nileesa Gautam, ${ }^{1}$ Krista F Huybrechts ${ }^{1,3}$
}

${ }^{1}$ Division of

Pharmacoepidemiology

and Pharmacoeconomics,

Department of Medicine,

Brigham and Women's Hospital

and Harvard Medical School,

1620 Tremont Street, Suite

3030, Boston, MA 02120, USA

${ }^{2}$ Department of Anesthesiology,

Perioperative and Pain Medicine, Brigham and Women's Hospital

and Harvard Medical School,

Boston, MA, USA

${ }^{3}$ Department of Epidemiology, Harvard T H Chan School of

Public Health, Boston, MA, USA

${ }^{4}$ Division of Maternal-Fetal

Medicine, Department of

Obstetrics and Gynecology,

Brigham and Women's Hospital,

Boston, MA, USA

Correspondence to: B T Bateman bbateman@bwh.harvard.edu (ORCID 0000-0001-5950-8683)

Additional material is published online only. To view please visit the journal online.

Cite this as: BMJ 2021;372:n102 http://dx.doi.org/10.1136/bmj.n102

Accepted: 11 December 2020

\section{ABSTRACT}

OBJECTIVE

To evaluate the risk of first trimester exposure to prescription opioids for major congenital malformations, previously reported to be associated with such exposure.

\section{DESIGN}

Population based cohort study.

SETTING

Nationwide sample of publicly and commercially insured pregnant women linked to their liveborn infants, nested in the Medicaid Analytic eXtract (MAX, 2000-14) and the MarketScan Research Database (MarketScan, 2003-15).

\section{PARTICIPANTS}

1602580 publicly insured (MAX) and 1177676 commercially insured (MarketScan) pregnant women with eligibility from at least three months before pregnancy to one month after delivery; infants with eligibility for at least three months after birth.

\section{INTERVENTIONS}

Use of prescription opioids was ascertained by requiring two or more dispensations of any opioid during the first trimester.

\section{MAIN OUTCOMES MEASURES}

Major malformations overall, cardiac malformations overall, ventricular septal defect, secundum atrial septal defect/patent foramen ovale, neural tube defect, clubfoot, and oral cleft, defined based on validated algorithms. Propensity score stratification was used to adjust for potential confounders and/ or proxies for confounders. Estimates from each database were combined using meta-analysis.

RESULTS

70447 (4.4\%) of 1602580 publicly insured and 12454 (1.1\%) of 1177676 commercially insured pregnant women had two or more dispensations of an opioid during the first trimester. Absolute risk of malformations overall was 41.0 (95\% confidence

\section{WHAT IS ALREADY KNOWN ON THIS TOPIC}

Pain is common during pregnancy, and opioid analgesics are routinely prescribed

Previous studies reported an association between exposure to opioids and certain congenital malformations, but data are few and conflicting

\section{WHAT THIS STUDY ADDS}

The findings suggest that prescription opioids used during the first trimester are not major teratogens, although clinicians and patients should be aware of the potential for a small increase in the risk of oral clefts associated with their use interval 39.5 to 42.5$)$ per 1000 pregnancies exposed to opioids versus 32.0 (31.7 to 32.3) per 1000 unexposed pregnancies in the MAX cohort, and 42.6 (39.0 to 46.1) and 37.3 (37.0 to 37.7) per 1000, respectively, in the MarketScan cohort. Pooled unadjusted relative risk estimates were raised for all outcomes but shifted substantially toward the null after adjustment; for malformations overall (relative risk 1.06, 95\% confidence interval 1.02 to 1.10$)$, cardiovascular malformations $(1.09,1.00$ to 1.18$)$, ventricular septal defect $(1.07,0.95$ to 1.21$)$, atrial septal defect/patent foramen ovale (1.04, 0.88 to 1.24$)$, neural tube defect $(0.82,0.53$ to 1.27$)$, and clubfoot $(1.06,0.88$ to 1.28$)$. The relative risk for oral clefts remained raised after adjustment (1.21, 0.98 to 1.50$)$, with a higher risk of cleft palate $(1.62,1.23$ to 2.14$)$.

\section{CONCLUSIONS}

Prescription opioids used in early pregnancy are not associated with a substantial increase in risk for most of the malformation types considered, although a small increase in the risk of oral clefts associated with their use is possible.

\section{Introduction}

Pain is common during pregnancy. In addition to the pain that affects women of reproductive age generally, pregnant women undergo a range of physiological changes, including increased ligamentous laxity and weight gain, which can cause or worsen a range of pain inducing conditions. ${ }^{1}$ Thus analgesics, including opioids, are drugs often used during pregnancy. ${ }^{2}$ Nationwide estimates in the United States suggest that approximately $22 \%$ of Medicaid beneficiaries ${ }^{3}$ and $14 \%$ of commercial insurance beneficiaries ${ }^{4}$ receive at least one prescription opioid during pregnancy. The frequency of opioid use during pregnancy in European countries, Canada, and Australia, although lower than in the US, is also substantial (around 5\% for most populations studied). ${ }^{5-10}$

Evidence for the teratogenicity of opioids from epidemiological studies is limited and conflicting. A systematic review from the Centers for Disease Control and Prevention in the US found that previous studies most often reported increases in the risk of congenital malformations overall, cardiovascular malformations overall, ventricular septal defect/atrial septal defect, spina bifida, oral cleft, and clubfoot. ${ }^{11}$ In view of the quality of the evidence considerable uncertainty remains about the association between opioids and congenital malformations. ${ }^{11} \mathrm{~A}$ teratogenic effect of opioids is biologically plausible as endogenous opioids 
are regulators of growth and development, raising concern that use of exogenous opioids at key times might disrupt normal developmental processes, giving rise to congenital malformations. ${ }^{12} 13$ The potential increase in the risk of birth defects associated with use of opioids has been highlighted by public health authorities. $^{14}$

Understanding this risk is important given the substantial number of women exposed during early pregnancy and the need to appraise the riskbenefit trade-off in the use of opioids for pain control during this period. Additionally, as about half of all pregnancies are unplanned, ${ }^{15}$ understanding the risk informs the safety of opioid use in women of reproductive age more generally. ${ }^{1617}$

In this study we assessed the association between exposure to prescription opioids during the first trimester and the risk of congenital malformations. We focused on types of malformation previously suggested to be associated with such exposure, while carefully controlling for confounding and other biases, using nationwide cohorts of publicly and commercially insured pregnant women.

\section{Methods}

Data source and study cohort

The study used pregnancy cohorts nested in the Medicaid Analytic eXtract (MAX), which includes data on healthcare use for Medicaid beneficiaries nationwide, for the years 2000 to 2014, and the IBM Health MarketScan Research Database (MarketScan), which includes data on healthcare use from a nationwide sample of commercially insured beneficiaries, for the years 2003 to 2015. The development of the MAX pregnancy cohort by linking maternal and infant claims records has been previously described $^{18}$; it has been used extensively to study the safety of drugs in pregnancy. ${ }^{19-26}$ The MarketScan pregnancy cohort was developed similarly, by linking maternal and infant claims. Both data sources include information on maternal demographics, diagnoses, and procedures received during inpatient, outpatient, or emergency department visits, and dispensed outpatient prescription drugs. The inclusion and exclusion criteria are described in appendix 1 and eFigures 1 and 2.

\section{Exposure to prescription opioids}

In the primary analysis, pregnancies were considered exposed if the mother filled at least two opioid prescriptions during the first trimester (first 90 days of pregnancy), which is the etiologically relevant exposure window for congenital malformations. eTable 1 lists the specific opioids included. We defined exposure based on two filled prescriptions on the assumption that if a woman refilled her prescription for opioids, she was probably consuming them. Pregnancies were considered unexposed if women did not fill an opioid prescription from 90 days before the date of the estimated last menstrual period through the end of the first trimester.

\section{Congenital malformations}

The primary study outcomes included congenital malformations overall, cardiovascular malformations overall, ventricular septal defect, secundum atrial septal defect/non-prematurity related patent foramen ovale, neural tube defect, clubfoot, and oral cleft (cleft lip or cleft palate, or both). These congenital malformations were selected as the primary study outcomes because they are the types most commonly found to have a significant association with opioid exposure in prior studies. ${ }^{11}$ Atrial septal defect and non-prematurity related patent foramen ovale were evaluated together as the International Classification of Disease, revision 9 (ICD-9) diagnostic codes do not distinguish between these malformations. The algorithms used to define malformations were based on diagnoses and procedure codes recorded in the infant record in the first three months after birth or in the maternal record in the first month after birth (because infant conditions might be recorded in the maternal claims before the infant's eligibility is processed; eTable 2). The positive predictive value of the algorithms used to identify the primary study outcomes was assessed by performing a validation study based on review of the medical chart (appendix 2). A range of additional malformations that might be associated with opioid exposure were also evaluated as secondary outcomes.

\section{Covariates}

We considered five groups of covariates as potential confounders or proxies for potential confounders, including indications for opioids, maternal demographic characteristics, chronic comorbidities, concomitant medication use, and general markers of the burden of illness (eTable 3). The potential indications for opioids included both acute and chronic pain conditions. Maternal demographic conditions assessed included age, calendar year of delivery, multiple gestation, and race/ethnicity (for Medicaid beneficiaries). Chronic maternal conditions were defined based on diagnostic codes recorded at any time during the three months before the last menstrual period until the end of the first trimester and included a range of medical and psychiatric conditions as well as use and abuse of non-opioid substances. Exposure to drugs that can act as proxies for maternal morbidity or its severity was based on filled prescriptions during the same assessment period as used for chronic conditions. Exposure to suspected teratogens was defined based on filled prescriptions during the first trimester. General markers of the burden of illness included the obstetric comorbidity index, ${ }^{27} 28$ number of non-opioid prescriptions, number of distinct diagnoses, and number of outpatient visits, admissions to hospital, and emergency visits (based on the three months before but not during pregnancy so that these measures are not affected by the early detection of pregnancy complications). These markers also control for confounding by access to medical care. 


\section{Analyses}

Analyses were conducted separately for the Medicaid and MarketScan cohorts. Unadjusted and adjusted risk estimates were further combined using fixed effects meta-analysis. ${ }^{29}$ Baseline characteristics of patients exposed and non-exposed to prescription opioids were compared using standardized differences. The absolute risks for the congenital malformations of interest were calculated, stratified by exposure to prescription opioids, and relative risks and 95\% confidence intervals were determined. We used prevalence of malformations at birth as a proxy for absolute risks for the outcome based on the assumption that, for most malformations, a relatively small proportion of fetuses with non-syndromic defects would die in utero or be terminated; given this, the actual risk might be slightly larger. ${ }^{30}$ For malformations with lower survival during pregnancy (eg, neural tube defects), the prevalence would underestimate the risk, but the prevalence ratio would still be valid, assuming that the termination of those pregnancies does not vary by maternal use of opioids within levels of the covariates adjusted for in the analyses.

Propensity score based methods were used to control for potential confounders or proxies for potential confounders. ${ }^{31}$ The propensity score for exposure to prescription opioids was estimated using a logistic regression model that included all covariates specified as potential confounders without further selection. After trimming observations from non-overlapping regions of the propensity score distribution, we created 50 strata based on the distribution among the exposed women. The unexposed women were weighted using the distribution of the exposed women among the propensity score strata to assess covariate balance after stratification. Adjusted relative risks were estimated by pooling the propensity score strata using the MantelHaenszel method.

A range of sensitivity analyses were conducted to evaluate the robustness of the results from our primary analyses. Firstly, we used high dimensional propensity scores to empirically identify 200 additional potential confounding variables or proxies for confounding variables. A high dimensional propensity score is an automated algorithm that has been shown to improve confounding control in some circumstances. ${ }^{32}$

Secondly, we conducted an analysis in which the reference group was redefined as women who had used prescription opioids before pregnancy but not during the first trimester (discontinuers), as the unmeasured conditions of these women might be more similar to those of women exposed to opioids during the first trimester.

Thirdly, to reduce the potential for exposure misclassification, we excluded women with opioid filling patterns that might suggest misuse or diversion. ${ }^{33} 34$

Fourthly, we restricted the analysis to women with dispensed folate supplements, as low folate intake is a risk factor for some of the malformations considered.
Fifthly, we redefined exposure to require that at least one dispensation of opioids was during the first trimester between six and 12 weeks after the last menstrual period, which is the critical time for some of the malformations considered (exposure during the late first trimester).

Sixthly, because some malformations might not be diagnosed shortly after birth, we extended the ascertainment of malformations to one year for infants who remained enrolled in our cohorts during this interval.

Seventhly, in a negative control analysis, we defined exposure to opioids based on dispensed prescriptions five to eight months after the last menstrual period, a time outside the etiologically relevant window for congenital malformations; a null finding associated with exposure in this window supports the notion that any observed association in the main analysis was not due to residual confounding.

Eighthly, because the cohorts were restricted to pregnancies ending in live birth, we assessed the impact of potentially different frequencies of nonlive births (that is, stillbirths, spontaneous abortions, terminations) in those exposed and not exposed to opioids within levels of covariates adjusted for in the analyses, using methods that have previously been described in detail (appendix 3). ${ }^{1921}$

Finally, for outcomes for which an increased risk was observed, to quantify the potential impact of residual confounding by factors incompletely measured in claims data, we assessed the extent of confounding necessary to fully explain the observed findings if there is no association using the target adjustment sensitivity analysis approach (appendix 4). ${ }^{35}$

We assessed opioid dispensing patterns during the first trimester (eFigure 3) and examined the effect of exposure to the most commonly used opioids, including hydrocodone, oxycodone, and codeine. We further performed analyses stratified by the amount of cumulative opioid exposure during the first trimester.

Analyses were conducted using SAS version 9.4 (SAS Institute). Precision of risk estimates are described using 95\% confidence intervals. Interpretation of the results was based on the strength of the adjusted risk ratio (regardless of whether the 95\% confidence interval includes the null); the degree to which the upper bound of the $95 \%$ confidence interval indicates low compatibility between the data and a strong adverse effect; and the consistency of the effect estimates across the sensitivity analyses that we conducted. No adjustments were made for multiple comparisons. The analyses of secondary endpoints should be considered as exploratory. The use of the deidentified database for research was approved by the institutional review board at Brigham and Women's Hospital.

\section{Patient and public involvement}

Members of the public were not included in the analysis owing to restriction on the use of the data included in the study and a lack of training in the use of these data. 


\section{Results}

\section{Cohort characteristics}

The MAX cohort consisted of 1602580 pregnancies, of which 70447 (4.4\%) were dispensed two or more opioid prescriptions during the first trimester. The MarketScan cohort consisted of 1177676 pregnancies, of which 12454 (1.1\%) were dispensed two or more opioids during the first trimester. About one third of all women exposed to opioids in both cohorts had prescriptions for hydrocodone only, followed by $13.9 \%$ (MAX) and 12.0\% (MarketScan) with prescriptions for hydrocodone and codeine, and 8.6\% (both cohorts) with prescriptions for codeine only (eFigure 3). Of the cohort, most women (MAX: 1135567 (84.4\%); MarketScan: $943928(89.3 \%))$ had one pregnancy, 171999 (12.8\%; MAX) and 105232 (10.0\%; MarketScan) had two pregnancies, and 37654 (2.8\%;
MAX) and 7558 (0.7\%; MarketScan) had three or more pregnancies. In both the MAX and MarketScan cohorts, substantial baseline differences were seen between women exposed to opioids and those who were not exposed. Women exposed to opioids were more likely to be diagnosed with pain conditions, to have comorbid psychiatric and medical conditions, to be dispensed other drugs in the first trimester, and to have higher measures of general comorbidity. For the MAX cohort, in which race/ethnicity is reported, women exposed to opioids were more likely to be white and less likely to be black or Hispanic (table 1, eTable 3). After propensity score stratification, all measured characteristics of the opioid exposed and unexposed women were well balanced in both cohorts with standardized differences less than 0.10 (table 2, eTable 4).

\begin{tabular}{|c|c|c|c|c|c|c|}
\hline & \multicolumn{3}{|c|}{ MAX 2000-14 } & \multicolumn{3}{|c|}{ MarketScan 2003-15 } \\
\hline & Exposed & Unexposed & $\begin{array}{l}\text { Standardized } \\
\text { difference }\end{array}$ & Exposed & Unexposed & $\begin{array}{l}\text { Standardized } \\
\text { difference }\end{array}$ \\
\hline Total & 70447 & 1532133 & - & 12454 & 1165222 & - \\
\hline Mean (SD) age & $26.03(5.46)$ & $24.27(5.98)$ & 0.31 & $32(4.85)$ & $31.97(4.6)$ & 0.01 \\
\hline \multicolumn{7}{|l|}{ Year of delivery: } \\
\hline $2000-02$ & $5234(7.43)$ & $162483(10.61)$ & -0.11 & NA & NA & NA \\
\hline 2003-05 & $15707(22.3)$ & $372573(24.31)$ & -0.05 & $1221(9.8)$ & $112153(9.63)$ & 0.01 \\
\hline $2006-10$ & $30531(43.34)$ & $617713(40.32)$ & 0.06 & $5724(45.96)$ & 485901 (41.7) & 0.09 \\
\hline $2011-15^{\star}$ & $18975(26.94)$ & $379364(24.76)$ & 0.05 & $5509(44.23)$ & $567168(48.68)$ & -0.09 \\
\hline \multicolumn{7}{|l|}{ Race/ethnicity: } \\
\hline White & $45328(64.34)$ & $543765(35.49)$ & 0.6 & $\mathrm{NA}$ & NA & NA \\
\hline Black & $15045(21.36)$ & $510311(33.31)$ & -0.27 & NA & NA & NA \\
\hline Hispanic & $5169(7.34)$ & $267363(17.45)$ & -0.31 & NA & NA & NA \\
\hline Other or unknown & $4905(6.96)$ & $210694(13.74)$ & -0.22 & NA & NA & NA \\
\hline \multicolumn{7}{|l|}{ Opioid indications: } \\
\hline Abdominal pain & $27837(39.51)$ & $235467(15.37)$ & 0.56 & $3049(24.48)$ & $68916(5.91)$ & 0.54 \\
\hline Back/neck pain & $29198(41.45)$ & $88306(5.76)$ & 0.93 & $4546(36.5)$ & $102311(8.78)$ & 0.7 \\
\hline Dental problems & $11710(16.62)$ & $22213(1.45)$ & 0.55 & $221(1.77)$ & $747(0.06)$ & 0.18 \\
\hline Fibromyalgia & $3385(4.81)$ & $7978(0.52)$ & 0.27 & $780(6.26)$ & $11189(0.96)$ & 0.29 \\
\hline Joint pain & $12318(17.49)$ & $40329(2.63)$ & 0.51 & $1638(13.15)$ & $30585(2.62)$ & 0.4 \\
\hline Migraine/headache & $16812(23.86)$ & $77038(5.03)$ & 0.56 & 2863 (22.99) & $38511(3.31)$ & 0.61 \\
\hline Orthopedic injury & $16459(23.36)$ & $58530(3.82)$ & 0.59 & $1943(15.6)$ & $41969(3.6)$ & 0.42 \\
\hline Surgery & $6663(9.46)$ & $19656(1.28)$ & 0.37 & $1374(11.03)$ & $7381(0.63)$ & 0.46 \\
\hline \multicolumn{7}{|l|}{ Maternal conditions: } \\
\hline Anxiety & $9468(13.44)$ & $43165(2.82)$ & 0.4 & $1199(9.63)$ & $32346(2.78)$ & 0.29 \\
\hline Depression & $10911(15.49)$ & $76216(4.97)$ & 0.35 & $1470(11.8)$ & $41210(3.54)$ & 0.31 \\
\hline Diabetes & $2478(3.52)$ & $21350(1.39)$ & 0.14 & $340(2.73)$ & $13374(1.15)$ & 0.11 \\
\hline Hypertension & $4518(6.41)$ & $27597(1.8)$ & 0.23 & $835(6.7)$ & $23801(2.04)$ & 0.23 \\
\hline \multicolumn{7}{|l|}{ Concomitant drugs: } \\
\hline Antidepressants & $21233(30.14)$ & $98446(6.43)$ & 0.64 & $3712(29.81)$ & $77348(6.64)$ & 0.63 \\
\hline Antiemetics & $27570(39.14)$ & $215914(14.09)$ & 0.59 & $5504(44.19)$ & $187701(16.11)$ & 0.64 \\
\hline Antihypertensives & $6192(8.79)$ & $32567(2.13)$ & 0.3 & $1316(10.57)$ & $30810(2.64)$ & 0.32 \\
\hline Benzodiazepines & $14600(20.72)$ & $24985(1.63)$ & 0.64 & $2971(23.86)$ & $31726(2.72)$ & 0.66 \\
\hline Antidiabetic drugs & $1402(1.99)$ & $11593(0.76)$ & 0.11 & $558(4.48)$ & $26712(2.29)$ & 0.12 \\
\hline Insulin & $1293(1.84)$ & $11102(0.72)$ & 0.1 & $265(2.13)$ & $10382(0.89)$ & 0.1 \\
\hline Psychostimulants & $1850(2.63)$ & $8038(0.52)$ & 0.17 & $527(4.23)$ & $7721(0.66)$ & 0.23 \\
\hline Suspected teratogens & $16477(23.39)$ & $137941(9)$ & 0.4 & $2098(16.85)$ & $79845(6.85)$ & 0.31 \\
\hline \multicolumn{7}{|c|}{ Mean (SD) general markers of comorbidity: } \\
\hline Obstetric comorbidity index & $0.52(0.97)$ & $0.24(0.64)$ & 0.34 & $0.72(1.04)$ & $0.49(0.82)$ & 0.25 \\
\hline Non-opioid prescription drugs & $3.82(3.49)$ & $1.12(1.79)$ & 0.97 & $3.7(3.43)$ & $1.2(1.82)$ & 0.91 \\
\hline Distinct diagnoses & $5.55(4.81)$ & $2.08(2.62)$ & 0.9 & $4.28(4.07)$ & $1.87(2.35)$ & 0.72 \\
\hline Emergency department visits & $0.96(1.73)$ & $0.2(0.61)$ & 0.59 & $0.24(0.8)$ & $0.03(0.23)$ & 0.35 \\
\hline Multiple gestation & $1142(1.62)$ & $19645(1.28)$ & 0.03 & $435(3.49)$ & $22949(1.97)$ & 0.09 \\
\hline
\end{tabular}

MarketScan=IBM Health MarketScan Research Database; MAX=Medicaid Analytic eXtract; NA=information not available for MarketScan

*2011-14 for MAX. 


\begin{tabular}{|c|c|c|c|c|c|c|}
\hline & \multicolumn{3}{|c|}{ MAX 2000-14 } & \multicolumn{3}{|c|}{ MarketScan 2003-15 } \\
\hline & Exposed & Unexposed & $\begin{array}{l}\text { Standardized } \\
\text { difference }\end{array}$ & Exposed & Unexposed & $\begin{array}{l}\text { Standardized } \\
\text { difference }\end{array}$ \\
\hline Total & 70074 & 1532118 & - & 12430 & 1152470 & - \\
\hline Mean (SD) age & $26.02(5.47)$ & $25.9(5.59)$ & 0.02 & $32(32.08)$ & $4.85(4.92)$ & -0.02 \\
\hline \multicolumn{7}{|l|}{ Year of delivery: } \\
\hline 2000-02 & $5228(7.46)$ & $120720(7.88)$ & -0.02 & NA & NA & NA \\
\hline $2003-05$ & $15660(22.35)$ & $352505(23.01)$ & -0.02 & $1221(9.82)$ & $112208(9.74)$ & 0.00 \\
\hline $2006-10$ & $30351(43.32)$ & $637860(41.63)$ & 0.03 & $5715(45.98)$ & $528656(45.88)$ & 0.00 \\
\hline $2011-15^{*}$ & $18835(26.87)$ & $421033(27.47)$ & -0.01 & $5494(44.19)$ & $511606(44.4)$ & 0.00 \\
\hline \multicolumn{7}{|l|}{ Race/ethnicity: } \\
\hline White & $45015(64.24)$ & $984729(64.27)$ & 0.00 & NA & NA & NA \\
\hline Black & $15015(21.43)$ & $330963(21.6)$ & 0.00 & NA & NA & NA \\
\hline Hispanic & $5160(7.36)$ & $111896(7.3)$ & 0.00 & NA & NA & NA \\
\hline Other or unknown & $4884(6.96)$ & $104530(6.82)$ & 0.01 & NA & NA & NA \\
\hline \multicolumn{7}{|l|}{ Opioid indications: } \\
\hline Abdominal pain & $27543(39.31)$ & $624298(40.75)$ & -0.03 & $3030(24.38)$ & $283167(24.57)$ & 0.00 \\
\hline Back/neck pain & $28850(41.17)$ & $629888(41.11)$ & 0.00 & $4523(36.39)$ & $434201(37.68)$ & -0.03 \\
\hline Dental problems & $11460(16.35)$ & $253711(16.56)$ & -0.01 & $216(1.74)$ & $19924(1.73)$ & 0.00 \\
\hline Fibromyalgia & $3282(4.68)$ & $77678(5.07)$ & -0.02 & $775(6.23)$ & $78666(6.83)$ & -0.02 \\
\hline Joint pain & $12093(17.26)$ & $270883(17.68)$ & -0.01 & $1626(13.08)$ & $151651(13.16)$ & 0.00 \\
\hline Migraine/headache & $16556(23.63)$ & $385438(25.16)$ & -0.04 & $2843(22.87)$ & $277320(24.06)$ & -0.03 \\
\hline Orthopedic injury & $16196(23.11)$ & $353848(23.1)$ & 0.00 & 1925 (15.49) & 178014 (15.45) & 0.00 \\
\hline Surgery & $6475(9.24)$ & $134270(8.76)$ & 0.02 & $1359(10.93)$ & $117529(10.2)$ & 0.02 \\
\hline \multicolumn{7}{|l|}{ Maternal conditions: } \\
\hline Anxiety & $9324(13.31)$ & 207851 (13.57) & -0.01 & $1188(9.56)$ & $112635(9.77)$ & -0.01 \\
\hline Depression & $10783(15.39)$ & $253206(16.53)$ & -0.03 & $1457(11.72)$ & $139548(12.11)$ & -0.01 \\
\hline Diabetes & $2452(3.5)$ & $56264(3.67)$ & -0.01 & $339(2.73)$ & $31067(2.7)$ & 0.00 \\
\hline Hypertension & $4439(6.33)$ & $98368(6.42)$ & 0.00 & $825(6.64)$ & 75969 (6.59) & 0.00 \\
\hline \multicolumn{7}{|l|}{ Concomitant drugs: } \\
\hline Antidepressants & $21002(29.97)$ & $482632(31.5)$ & -0.03 & $3697(29.74)$ & $342963(29.76)$ & 0.00 \\
\hline Antiemetics & $27289(38.94)$ & $593935(38.77)$ & 0.00 & $5481(44.09)$ & $513389(44.55)$ & -0.01 \\
\hline Antihypertensives & $6108(8.72)$ & $137786(8.99)$ & -0.01 & $1305(10.5)$ & $117523(10.2)$ & 0.01 \\
\hline Benzodiazepines & $14340(20.46)$ & $307909(20.1)$ & 0.01 & $2952(23.75)$ & $264276(22.93)$ & 0.02 \\
\hline Antidiabetic drugs & $1390(1.98)$ & $32018(2.09)$ & -0.01 & $556(4.47)$ & $52196(4.53)$ & 0.00 \\
\hline Insulin & $1280(1.83)$ & $29833(1.95)$ & -0.01 & $265(2.13)$ & $25598(2.22)$ & -0.01 \\
\hline Psychostimulants & $1819(2.6)$ & $42073(2.75)$ & -0.01 & $522(4.2)$ & 44927 (3.9) & 0.02 \\
\hline Suspected teratogens & $16309(23.27)$ & $357238(23.32)$ & 0.00 & $2094(16.85)$ & $195552(16.97)$ & 0.00 \\
\hline \multicolumn{7}{|c|}{ Mean (SD) general markers of comorbidity: } \\
\hline Obstetric comorbidity index & $0.52(0.96)$ & $0.53(0.97)$ & -0.01 & $0.72(0.74)$ & $1.04(1.04)$ & -0.02 \\
\hline Non-opioid prescription drugs & $3.79(3.46)$ & $3.64(3.24)$ & 0.04 & $3.69(3.59)$ & $3.42(3.14)$ & 0.03 \\
\hline Distinct diagnoses & $5.48(4.7)$ & $5.71(4.92)$ & -0.05 & $4.25(4.6)$ & $4.02(4.44)$ & -0.08 \\
\hline Emergency department visits & $0.93(1.66)$ & $0.85(1.53)$ & 0.05 & $0.23(0.2)$ & $0.76(0.58)$ & 0.06 \\
\hline Multiple gestation & $1135(1.62)$ & $22917(1.5)$ & 0.01 & $435(3.5)$ & $42343(3.67)$ & -0.01 \\
\hline
\end{tabular}

*2011-14 for MAX.

\section{Risk of congenital malformations}

Absolute risk estimates for the primary outcomes in the opioid exposed versus unexposed patients in both cohorts are shown in eTable 5 . The pooled unadjusted relative risk estimates were raised for all primary study outcomes, including congenital malformations overall, cardiovascular malformations overall, ventricular septal defect, atrial septal defect/patent foramen ovale, neural tube defect, clubfoot, and oral cleft (fig 1 and fig 2).

Relative risk estimates shifted substantially toward the null after adjustment for all measured covariates using propensity score stratification. Pooled estimates did not suggest a substantially increased risk for congenital malformations overall (relative risk 1.06, 95\% confidence interval 1.02 to 1.10 ), cardiovascular malformations overall $(1.09,1.00$ to 1.18$)$, ventricular septal defect $(1.07,0.95$ to 1.21$)$, atrial septal defect/ patent foramen ovale (1.04, 0.88 to 1.24$)$, neural tube defect $(0.82,0.53$ to 1.27$)$, or clubfoot $(1.06,0.88$ to 1.28). In contrast, the pooled risk estimate for oral cleft remained high after adjustment (1.21, 0.98 to 1.50). This increase was explained by a higher risk of cleft palate (1.62, 1.23 to 2.14$)$; estimates were close to the null for cleft lip (1.02, 0.69 to 1.51) and cleft palate with cleft lip (1.08, 0.79 to 1.47; eFigure 4).

Sensitivity, subgroup, and exploratory analyses Sensitivity analyses to deal with the potential for unmeasured confounding (high dimensional propensity score adjustment and discontinuers as reference group) yielded estimates generally consistent with those from the main analysis, accounting for the width of the confidence intervals. Similarly, generally 


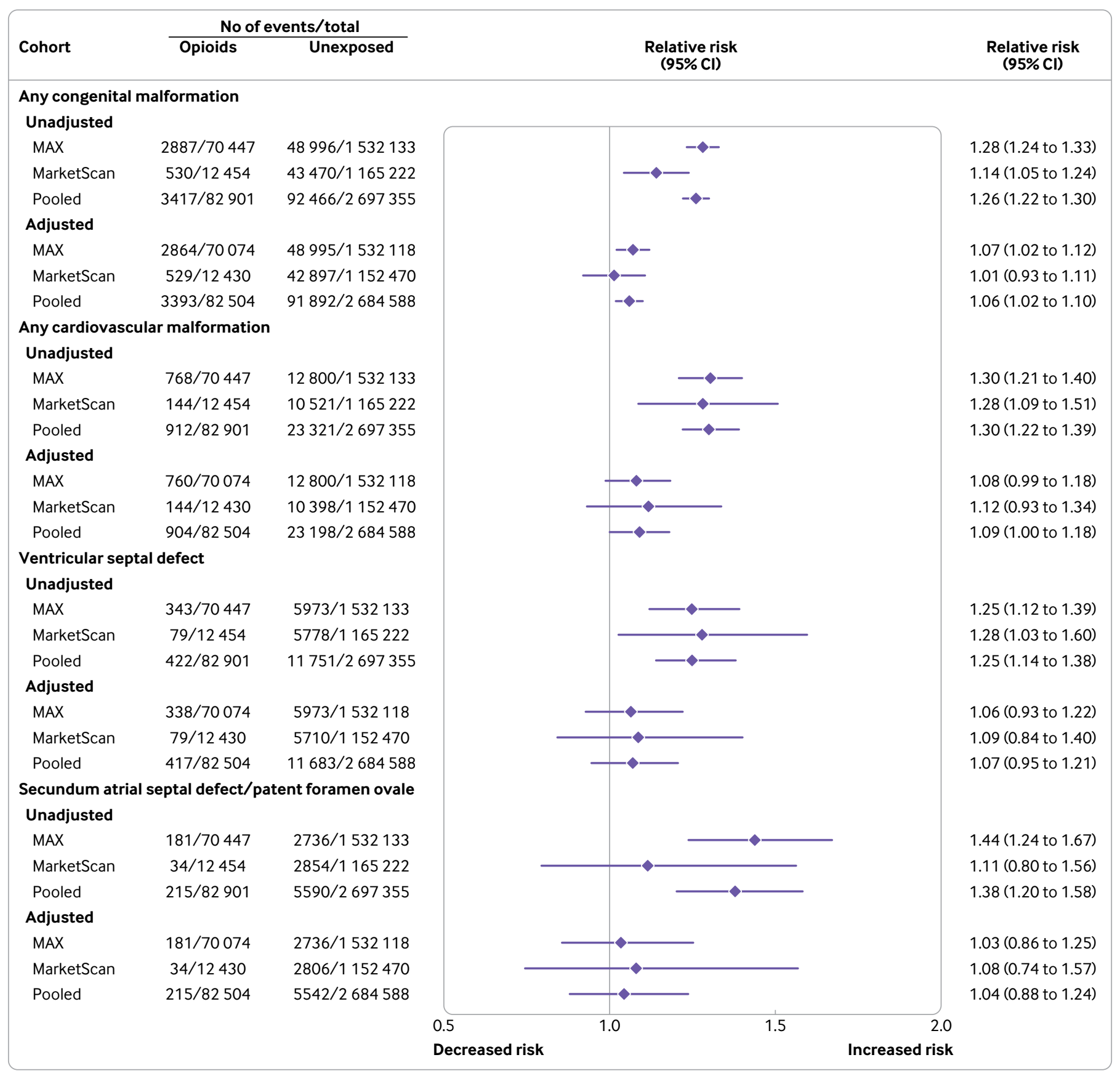

Fig 1 | Risk of congenital malformations (primary outcomes) in infants after exposure to opioids during the first trimester: main analyses (unadjusted and propensity score stratified). MarketScan=IBM Health MarketScan Research Database; MAX=Medicaid Analytic eXtract

consistent results were seen when women with aberrant opioid filling patterns were excluded, when the cohort was restricted to women with dispensed prescriptions for folate supplements, and when the outcome assessment period was extended to 365 days after birth. Redefining exposure based on late first trimester dispensing slightly strengthened the association for oral clefts (relative risk 1.32, 95\% confidence interval 1.03 to 1.68 ), but did not substantially shift the estimates of the other malformations. In the negative control analysis, no increased risk was observed for oral clefts, and the estimates shifted substantially toward the null for cleft palate (fig 3, fig 4, eFigure 5, eTables 6 and 7).

Under the strongest assumptions tested for the potential impact of selection bias due to restriction to live births, the relative risk estimates would remain below 1.30 for the malformations of interest (appendix 3). Oral clefts were not included in this analysis as nonsyndromic oral clefts do not result in fetal death and are rarely a reason for terminations.

For oral cleft, and specifically cleft palate, we assessed the extent of residual confounding necessary to fully explain the observed adjusted association 


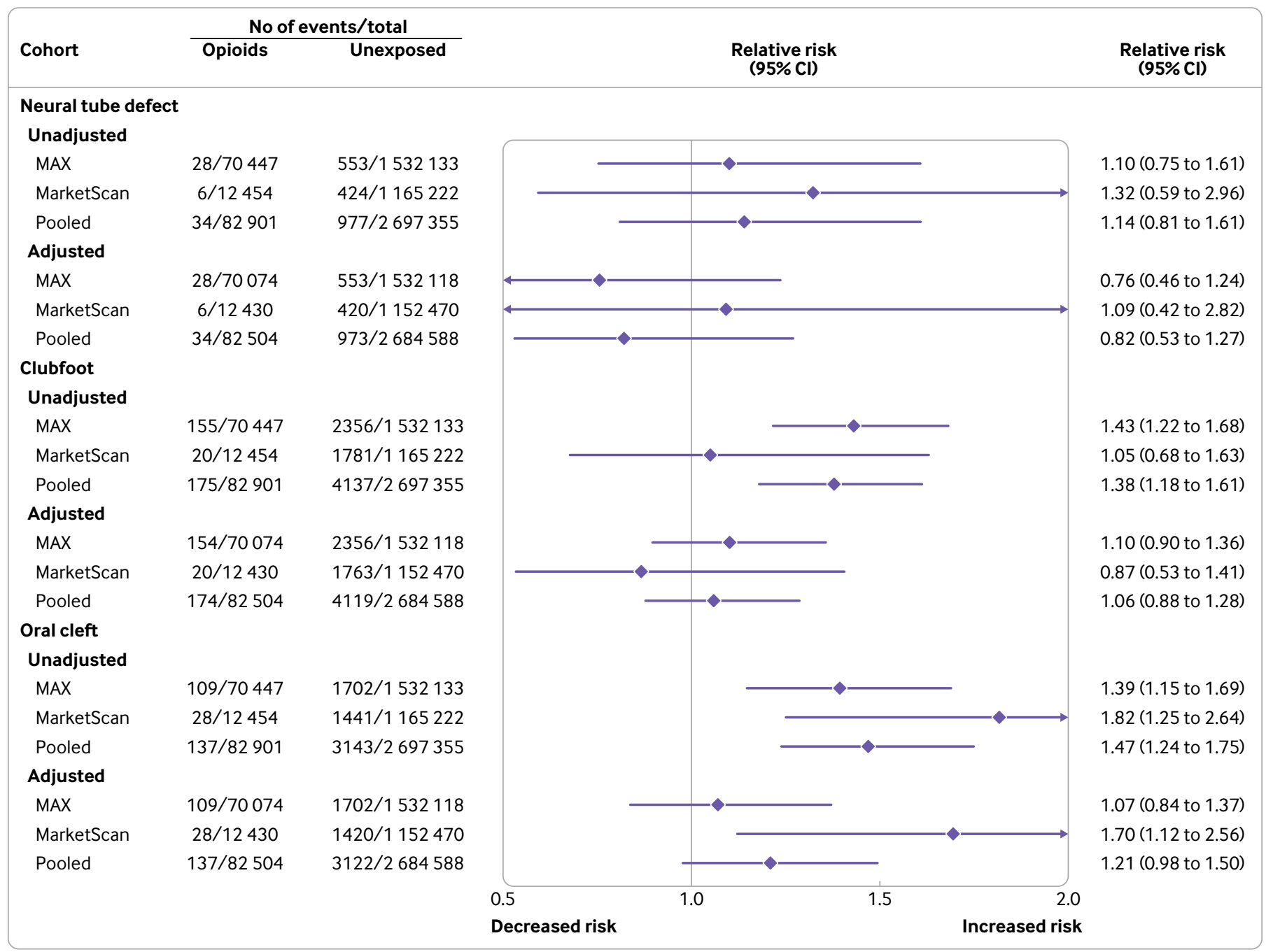

Fig 2 | Risk of congenital malformations (primary outcomes) in infants after exposure to opioids during the first trimester: main analyses (unadjusted and propensity score stratified). MarketScan=IBM Health MarketScan Research Database; MAX=Medicaid Analytic eXtract

if there is none (appendix 4). For an unmeasured confounder present in 10 or 20 percent of the population, relative risks of 2.5 or more linking the hypothetical confounder to both opioid exposure and oral cleft would be needed to fully explain the observed association. For cleft palate, relative risks of 4 or more would be necessary.

The risk of the primary outcomes was evaluated for each of the most commonly used specific opioids (fig 5, fig 6, eFigure 6, eTables 6 and 7). Adjusted estimates were consistent with those from the evaluation of opioids overall, although confidence intervals were, in some instances, wide. No evidence of increasing risk with higher cumulative opioid exposure was found for any of the primary outcomes, with the possible exception of oral clefts and specifically, cleft palates, for which the point risk estimate for the group receiving the lowest dose was near the null, whereas it was raised for each of the other three dose groups.

We evaluated the associations between prescription opioids and a range of specific (secondary) malformations in exploratory analyses (eFigures 7 and 8,
eTable 8). In the context of multiple comparisons, a small increase was seen in the point estimates for gastroschisis and anomalous pulmonary venous return, but confidence intervals were wide and included the null. We further observed an approximately 40 percent increase in the risk of hydrocephaly and of persistent pulmonary hypertension of the newborn.

\section{Discussion}

Principal findings

In this study, which included more than 82000 pregnant women exposed to prescription opioids during the first trimester drawn from approximately 2.7 million pregnancies, no substantial increase was seen in the risk for congenital malformations overall, cardiovascular malformations overall, ventricular septal defect, atrial septal defect/patent foramen ovale, neural tube defect, or clubfoot with in utero opioid exposure. The upper bound of the $95 \%$ confidence interval from the pooled adjusted estimates in the main analysis excluded a more than 30 percent increase in the risk for these malformations. Although 


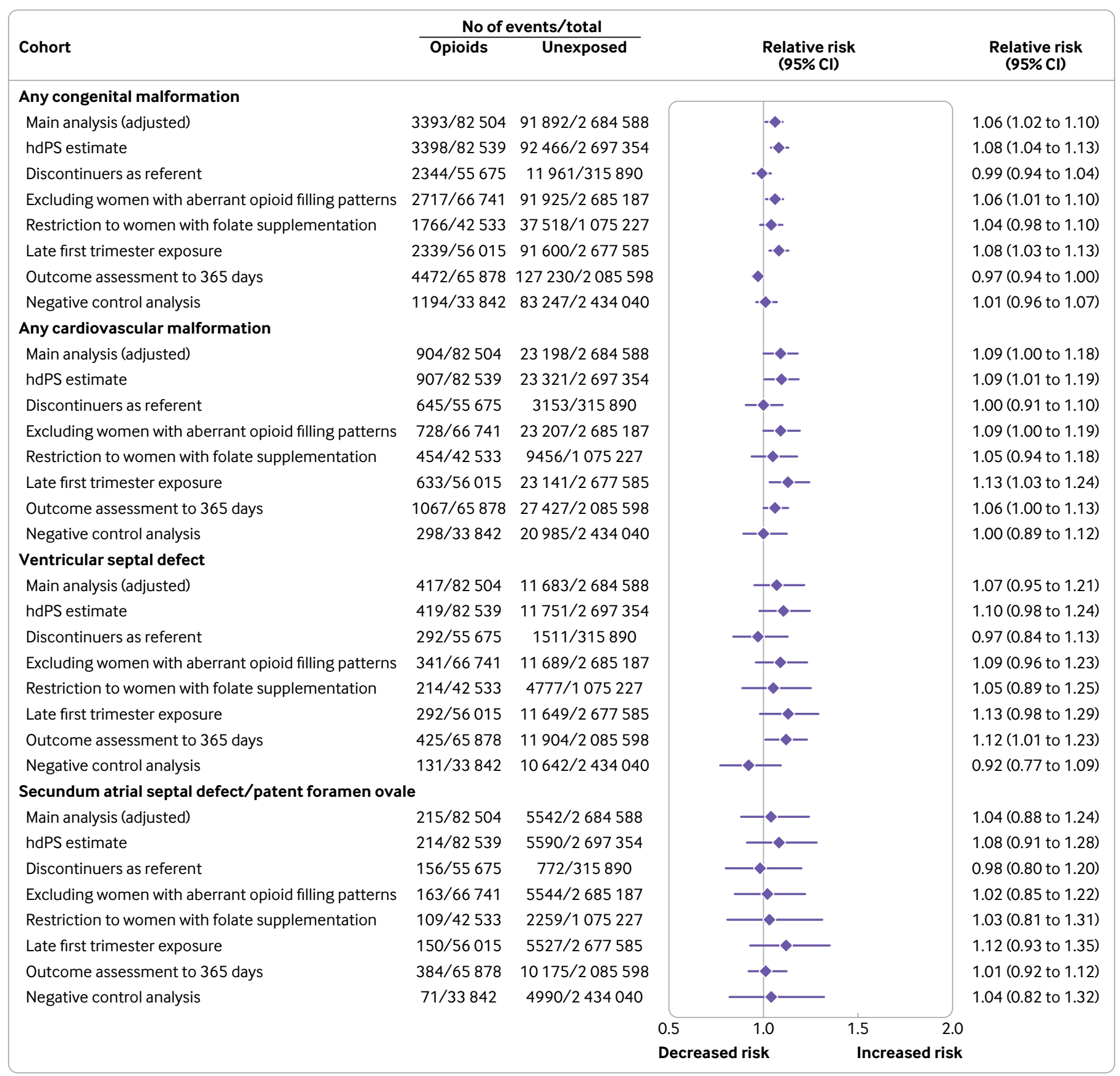

Fig 3 | Risk of congenital malformations (primary outcomes) in infants after exposure to opioids during the first trimester: sensitivity analyses (pooled estimates, propensity score stratified). Aberrant opioid filling patterns were defined based on an average of $>120 \mathrm{mg}$ oral morphine milligram equivalents for 90 or more consecutive days or use of more than three pharmacies or more than three prescribers for opioid prescriptions between three months before pregnancy and the end of the first trimester. hdPS=high dimensional propensity score

point estimates for risk were slightly raised for some of these outcomes (eg, relative risk of 1.06 for any congenital malformations and 1.09 for cardiovascular malformations overall), given the observational nature of the study, these small increases should probably not be considered clinically meaningful.

In contrast, the risk for oral clefts was increased, which was attributable to an increase in the risk for cleft palate. This increase in risk corresponds to four to five additional cases of cleft palate per 10000 pregnancies exposed to opioids in the first trimester.
The consistency of the finding for oral clefts across multiple sensitivity analyses, and the null finding when exposure was based on dispensation of the drug outside of the etiologically relevant window, reinforce the suggestion that the effect is unlikely to be due to residual confounding.

\section{Comparison with other studies}

Our analyses expand the available evidence for the safety of opioids in pregnancy. Based on a systematic review, 12 case-control and 18 cohort 


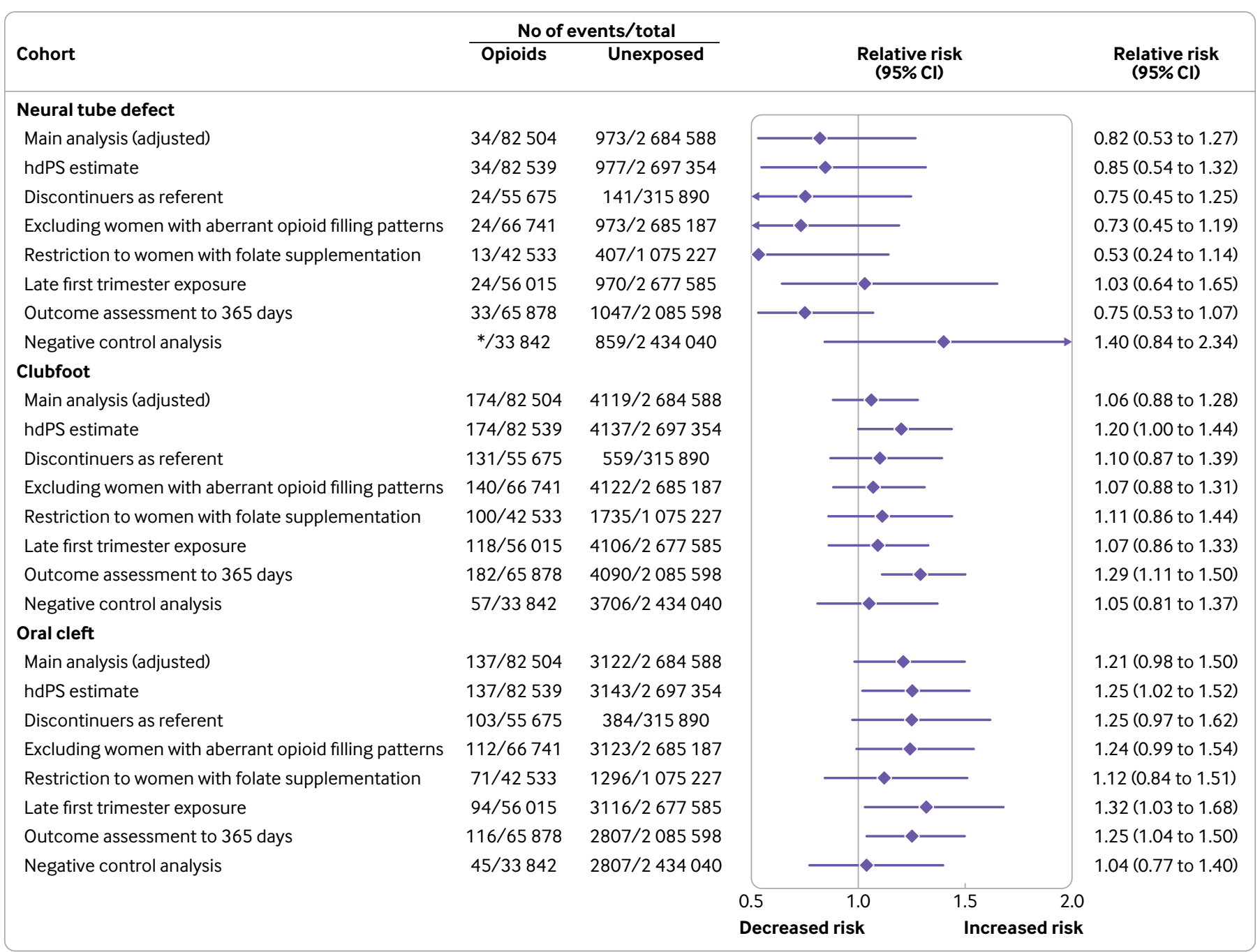

Fig 4 | Risk of congenital malformations (primary outcomes) in infants after exposure to opioids during the first trimester: sensitivity analyses (pooled estimates, propensity score stratified). ${ }^{*}$ Cell size less than 11 for the MAX cohort. Numbers suppressed in accordance with the CMS cell size suppression policy. Aberrant opioid filling patterns were defined based on an average of $>120 \mathrm{mg}$ oral morphine milligram equivalents for 90 or more consecutive days or use of more than three pharmacies or more than three prescribers for opioid prescriptions between three months before pregnancy and the end of the first trimester. CMS=Centers for Medicare and Medicaid Services; hdPS=high dimensional propensity score; MAX=Medicaid Analytic eXtract

studies have previously measured the association between opioid exposure in pregnancy and congenital malformations. ${ }^{11}$ Of these 30 studies, 17 reported significant associations with at least one type of malformation. The possibility of confounding bias, outcome and exposure misclassification, and recall bias could not be excluded for some studies, however, and combined with limited power led to uncertainty about the teratogenic potential of opioids. ${ }^{11}$ The number of opioid exposed pregnancies included in our study is about 10 -fold larger than in any cohort study published to date, ${ }^{11}$ allowing relatively precise risk estimates for specific malformation types, while carefully controlling for a large number of potential confounders. The analyses were based on data from nationwide cohorts of both Medicaid and commercially insured pregnant women, making the cohorts broadly representative of the entire obstetric population in the US. Furthermore, outcomes were defined using algorithms with a high positive predictive value. Finally, the large cohort size and the information available about filled prescriptions allowed us to examine the most commonly used opioid types individually and to assess the effect of dose.

Previous studies identified in the Centers for Disease Control and Prevention systematic review which showed an increase in the risk for oral clefts, are older (completed in the 1970s and 1980s). ${ }^{11}$ More recent case-control data also point to a potential increase in the risk for oral clefts; data from the National Birth Defects Prevention Study reported increased point estimates for cleft palate and cleft lip with cleft palate, albeit with wide confidence intervals that intersected the null (cleft palate: adjusted odds ratio 1.3, 95\% confidence interval 0.84 to 2.0; cleft lip with cleft palate: $1.4,0.96$ to 2.1 ), but not isolated cleft lip (cleft lip without cleft palate: $0.68,0.34$ to 1.3$).{ }^{12}$ In our analysis, the point estimates were near the null 


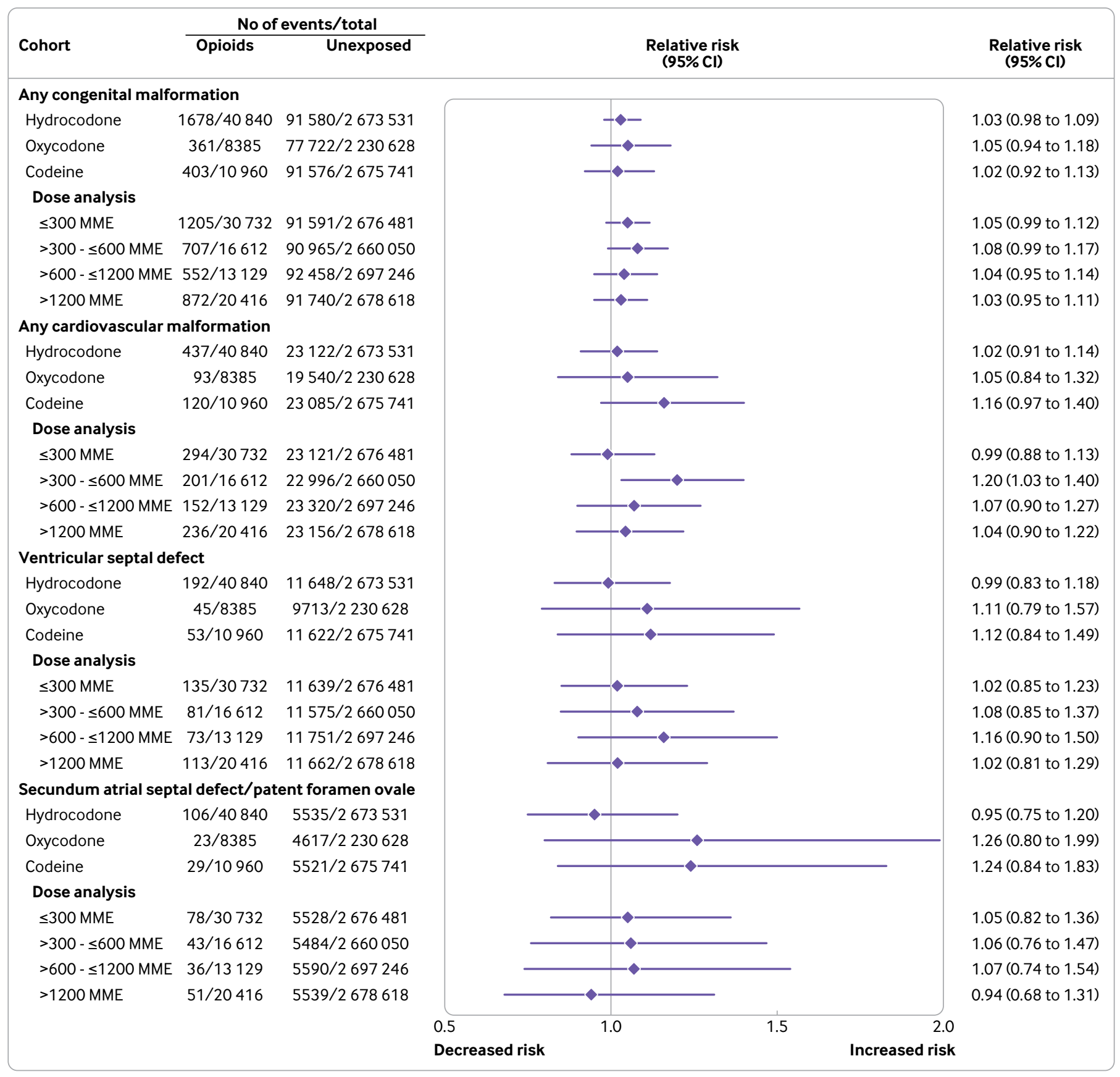

Fig 5 | Risk of congenital malformations (primary outcomes) in infants after exposure to opioids during the first trimester: subgroup analyses (pooled estimates, propensity score stratified). $M M E=$ morphine milligram equivalent

for isolated cleft lip and cleft lip with cleft palate, but raised for cleft palate.

\section{Limitations}

Our study is subject to certain limitations inherent in its design. Exposure is defined based on filled prescriptions, which does not necessarily indicate use. To minimize the risk of exposure misclassification, we required that women filled at least two prescriptions during the first trimester to be classified as exposed on the assumption that if a woman refills her opioid prescription, she is taking it as prescribed.
As with all observational studies, residual confounding is a potential concern. However, we adjust for a large number of indications for opioids, as well as co-exposures to drugs and medical and obstetric conditions that might be associated with opioid exposure.

Further, we observed null or near null associations for most of the primary study outcomes; confounding resulting in downward bias associated with opioid exposure is highly unlikely, which allays this concern. The observed increase in the risk of oral clefts (and cleft palate, in particular) is of greater concern. 


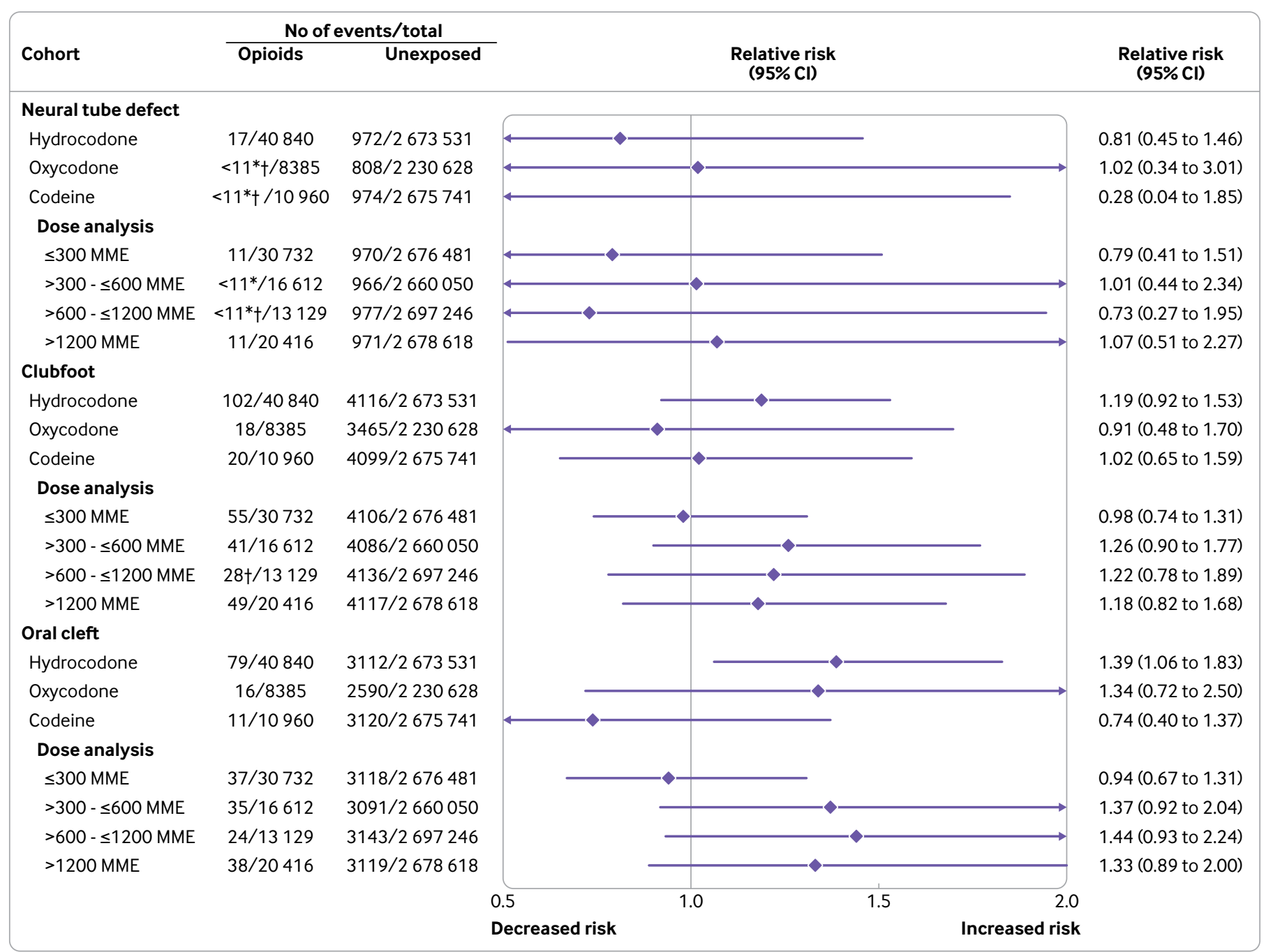

Fig 6 | Risk of congenital malformations (primary outcomes) in infants after exposure to opioids during the first trimester: subgroup analyses (pooled estimates, propensity score stratified). *Cell size less than 11 for the MAX cohort. Numbers are suppressed in accordance with the CMS cell size suppression policy. tResults presented here are not pooled but represent results in the MAX cohort as there were no outcomes among infants exposed in the MarketScan cohort. CMS=Centers for Medicare and Medicaid Services; MarketScan=IBM Health MarketScan Research Database; $M A X=$ Medicaid Analytic eXtract; $M M E=$ morphine milligram equivalent

Yet, given the null/near null findings for the other malformations, residual confounding for one outcome but none of the others seems unlikely. Furthermore, the increase in risk of oral clefts persisted across multiple sensitivity analyses designed to deal with residual confounding, including the use of high dimensional propensity score analysis and an alternative reference group of opioid discontinuers. Additionally, no increase in the risk of oral clefts was seen in a negative control analysis in which the association with opioid exposure outside the etiologically relevant window was assessed, providing indirect evidence of no substantial residual confounding. As indicated by the target adjustment sensitivity analysis, the strength of an association between an unmeasured confounder and both opioid exposure and oral clefts would need to be unrealistically high to fully explain the observed association based on residual confounding.
The analysis was based on pregnancies resulting in live births, which might introduce selection bias. Formal measurement of the potential for selection bias, however, suggests that in the range of plausible differences in the proportion of non-live born pregnancies to opioid users versus non-users the effect of such selection bias on risk estimates is likely to be small.

Finally, although the study population included pregnant Medicaid and commercial insurance beneficiaries, the characteristics of this patient population are not expected to affect the biological associations studied and, therefore, the findings should be generalizable to other populations.

\section{Conclusions}

Overall, our findings suggest that prescription opioids used in early pregnancy are not associated with a substantial increase in risk for most of the malformation types considered, although clinicians should be aware 
of the potential for a small increase in the risk of oral clefts and counsel patients about this risk. The results inform the selection of analgesic drugs for women who are pregnant and women of reproductive age who might inadvertently become pregnant.

Contributors: BTB, SH-D, and KFH contributed to all the aspects of this study. HM was involved in preparation of analytic datasets and designing the study. KJG, NG, RJD, and YZ were involved in designing the study and preparation of the final manuscript. LS was involved in preparation of analytic datasets, designing the study, and preparation of the final manuscript. All authors had full access to all of the data (including statistical reports and tables) in the study and take responsibility for the integrity of the data and the accuracy of the data analysis. BTB and KFH are the guarantors. The corresponding author attests that all listed authors meet authorship criteria and that no others meeting the criteria have been omitted.

Funding: This study was supported by grant R01-DA044293 from the National Institute on Drug Abuse. The funding source had no role in the study design; in the collection, analysis, and interpretation of the data; in the writing of the report; and in the decision to submit the article for publication.

Competing interests: All authors have completed the ICMJE uniform disclosure form at www.icmje.org/coi_disclosure.pdf and declare: support from the National Institute on Drug Abuse for the submitted work; no financial relationships with any organization that might have an interest in the submitted work in the previous three years; no other relationships or activities that could appear to have influenced the submitted work. KFH reports grants from the National Institute of Health and the US Food and Drug Administration during the conduct of the study and grants to her institution from Eli Lilly, Takeda, and GlaxoSmithKline outside the submitted work. SH-D reports grants from the National Institutes of Health and the US Food and Drug Administration during the conduct of the study; grants to her institution from Eli Lilly, Takeda, and GlaxoSmithKline outside the submitted work; and being an adviser for the Antipsychotics Pregnancy Registry and epidemiologist for the North American Antiepileptics Pregnancy Registry, both at Massachusetts General Hospital. RJD reports research support from Novartis, Bayer, and Vertex for unrelated projects. KJG reports having consulted for BillionToOne, Quest Diagnostics, Inc, and Illumina, Inc on unrelated topics. BTB reports grants from the National Institutes of Health and the US Food and Drug Administration during the conduct of the study and grants to his institution from Eli Lilly, Takeda, GlaxoSmithKline, Baxalta, and Pacira outside the submitted work; he was a consultant to Aetion, Inc and the Alosa Foundation. All other authors declare no other relationships or activities that could appear to have influenced the submitted work

Ethical approval: The use of these deidentified databases for research was approved by the institutional review board of the Brigham and Women's Hospital, Boston, MA, and a data use agreement was in place.

Data sharing: No additional data available.

BTB and KFH affirm that the manuscript is an honest, accurate, and transparent account of the study being reported; that no important aspects of the study have been omitted; and that any discrepancies from the study as originally planned (and, if relevant, registered) have been explained.

Dissemination to participants and related patient and public communities: The study results will be disseminated to the public through media releases and social media.

Provenenance and peer review: Not commissioned; externally peer reviewed.

This is an Open Access article distributed in accordance with the Creative Commons Attribution Non Commercial (CC BY-NC 4.0) license, which permits others to distribute, remix, adapt, build upon this work non-commercially, and license their derivative works on different terms, provided the original work is properly cited and the use is noncommercial. See: http://creativecommons.org/licenses/by-nc/4.0/.

1 Casagrande D, Gugala Z, Clark SM, Lindsey RW. Low back pain and pelvic girdle pain in pregnancy. J Am Acad Orthop Surg 2015;23:539-49. doi:10.5435/JAAOS-D-14-00248

2 Palmsten K, Hernández-Díaz S, Chambers CD, et al. The most commonly dispensed prescription medications among pregnant women enrolled in the US Medicaid program. Obstet Gynecol 2015;126:465-73. doi:10.1097/ AOG.0000000000000982

3 Desai RJ, Hernandez-Diaz S, Bateman BT, Huybrechts KF. Increase in prescription opioid use during pregnancy among Medicaid-enrolled women. Obstet Gynecol 2014;123:997-1002. doi:10.1097/ AOG 0000000000000208

4 Bateman BT, Hernandez-Diaz S, Rathmell JP, et al. Patterns of opioid utilization in pregnancy in a large cohort of commercial insurance beneficiaries in the United States. Anesthesiology 2014:120:121624. doi:10.1097/ALN.0000000000000172

5 Sujan AC, Quinn PD, Rickert ME, et al. Maternal prescribed opioid analgesic use during pregnancy and associations with adverse birth outcomes: a population-based study. PLoS Med 2019;16:e1002980. doi:10.1371/journal.pmed.1002980

6 Engeland A, Bjørge T, Klungsøyr K, Hjellvik V, Skurtveit S, Furu K. Trends in prescription drug use during pregnancy and postpartum in Norway, 2005 to 2015. Pharmacoepidemiol Drug Saf 2018;27:995 1004. doi: $10.1002 / p d s .4577$

7 Miller AM, Sanderson K, Bruno RB, Breslin M, Neil AL. Chronic pain, pain severity and analgesia use in Australian women of reproductive age. Women Birth 2019;32:e272-8. doi:10.1016/j. wombi.2018.06.013

8 Daw JR, Mintzes B, Law MR, Hanley GE, Morgan SG. Prescription drug use in pregnancy: a retrospective, population-based study in British Columbia, Canada (2001-2006). Clin Ther 2012;34:239-49.e2 doi:10.1016/j.clinthera.2011.11.025

9 Irvine L, Flynn RW, Libby G, Crombie IK, Evans JM. Drugs dispensed in primary care during pregnancy: a record-linkage analysis in Tayside, Scotland. Drug Saf 2010;33:593-604. doi:10.2165/11532330$000000000-00000$

10 Handal M, Engeland A, Rønning M, Skurtveit S, Furu K. Use of prescribed opioid analgesics and co-medication with benzodiazepines in women before, during, and after pregnancy: a population-based cohort study. Eur J Clin Pharmacol 2011;67:95360. doi:10.1007/s00228-011-1030-7

11 Lind JN, Interrante JD, Ailes EC, et al. Maternal use of opioids during pregnancy and congenital malformations: a systematic review. Pediatrics 2017;139:e20164131. doi:10.1542/peds.2016-4131

12 Broussard CS, Rasmussen SA, Reefhuis J, et al, National Birth Defects Prevention Study. Maternal treatment with opioid analgesics and risk for birth defects. Am J Obstet Gynecol 2011;204:314.e1-11. doi:10.1016/j.ajog.2010.12.039

13 Zagon IS, Wu Y, McLaughlin PJ. Opioid growth factor and organ development in rat and human embryos. Brain Res 1999;839:31322. doi:10.1016/S0006-8993(99)01753-9

14 Centers for Disease Control and Prevention. About opioid use during pregnancy. 2020. https://www.cdc.gov/pregnancy/opioids/basics. html.

15 Finer LB, Zolna MR. Declines in unintended pregnancy in the United States, 2008-2011. N Engl J Med 2016;374:843-52. doi:10.1056/ NEJMsa 1506575

16 Gallagher BK, Shin Y, Roohan P. Opioid prescriptions among women of reproductive age enrolled in Medicaid - New York, 2008-2013. MMWR Morb Mortal Wkly Rep 2016;65:415-7. doi:10.15585/mmwr. $\mathrm{mm} 6516 \mathrm{a} 2$

17 Ailes EC, Dawson AL, Lind IN, et al, Centers for Disease Control and Prevention (CDC). Opioid prescription claims among women of reproductive age--United States, 2008-2012. MMWR Morb Mortal Wkly Rep 2015;64:37-41

18 Palmsten K, Huybrechts KF, Mogun H, et al. Harnessing the Medicaid Analytic eXtract (MAX) to evaluate medications in pregnancy: design considerations. PLoS One 2013;8:e67405. doi:10.1371/journal. pone.0067405

19 Huybrechts KF, Palmsten K, Avorn J, et al. Antidepressant use in pregnancy and the risk of cardiac defects. $N$ Engl J Med 2014:370:2397-407. doi:10.1056/NEJMoa1312828

20 Patorno E, Huybrechts KF, Bateman BT, et al. Lithium use in pregnancy and the risk of cardiac malformations. N Engl J Med 2017:376:2245-54. doi:10.1056/NEIMoa1612222

21 Bateman BT, Hernandez-Diaz S, Fischer MA, et al. Statins and congenital malformations: cohort study. BMJ 2015;350:h1035. doi:10.1136/bmj.h1035

22 Desai RJ, Huybrechts KF, Hernandez-Diaz S, et al. Exposure to prescription opioid analgesics in utero and risk of neonatal abstinence syndrome: population based cohort study. BMJ 2015:350:h2102. doi:10.1136/bmi.h2102

23 Huybrechts KF, Bateman BT, Palmsten K, et al. Antidepressant use late in pregnancy and risk of persistent pulmonary hypertension of the newborn. JAMA 2015;313:2142-51. doi:10.1001/ jama.2015.5605

24 Huybrechts KF, Hernández-Díaz S, Patorno E, et al. Antipsychotic use in pregnancy and the risk for congenital malformations. JAMA Psychiatry 2016;73:938-46. doi:10.1001/ jamapsychiatry.2016.1520 
25 Huybrechts KF, Hernández-Díaz S, Straub L, et al. Association of maternal first-trimester ondansetron use with cardiac malformations and oral clefts in offspring. JAMA 2018:320:2429-37. doi:10.1001/ jama.2018.18307

26 Huybrechts KF, Hernandez-Diaz S, Straub L, et al. Intravenous ondansetron in pregnancy and risk of congenital malformations. JAMA 2020;323:372-4. doi:10.1001/jama.2019.18587

27 Bateman BT, Mhyre JM, Hernandez-Diaz S, et al. Development of a comorbidity index for use in obstetric patients. Obstet Gynecol 2013;122:957-65. doi:10.1097/ AOG.0b013e3182a603bb

28 Metcalfe A, Lix LM, Johnson JA, et al. Validation of an obstetric comorbidity index in an external population. BJOG 2015;122:174855. doi:10.1111/1471-0528.13254

29 DerSimonian R, Laird N. Meta-analysis in clinical trials. Control Clin Trials 1986:7:177-88 doi:10.1016/0197-2456(86)90046-2

30 Svensson E, Ehrenstein V, Nørgaard M, et al. Estimating the proportion of all observed birth defects occurring in pregnancies terminated by a second-trimester abortion. Epidemiology 2014;25:866-71. doi:10.1097/ EDE.0000000000000163

31 Desai RJ, Rothman KJ, Bateman BT, Hernandez-Diaz S, Huybrechts KF. A propensity-score-based fine stratification approach for confounding adjustment when exposure is infrequent. Epidemiology 2017;28:249-57. doi:10.1097/ EDE.0000000000000595

32 Schneeweiss S, Rassen JA, Glynn RJ, Avorn J, Mogun H, Brookhart MA. High-dimensional propensity score adjustment in studies of treatment effects using health care claims data. Epidemiology 2009;20:512-22. doi:10.1097/ EDE.0b013e3181a663cc

33 Tudor CG. Memorandum: Medicare Part D overutilization monitoring system. 2013. https://www.cms.gov/Medicare/Prescription-DrugCoverage/PrescriptionDrugCovContra/Downloads/HPMS-memoMedicare-Part-D-Overutilization-Monitoring-System-07-05-13-.pdf.

34 Rough K, Huybrechts KF, Hernandez-Diaz S, Desai RJ, Patorno E, Bateman BT. Using prescription claims to detect aberrant behaviors with opioids: comparison and validation of 5 algorithms. Pharmacoepidemiol Drug Saf 2019;28:62-9. doi:10.1002/ pds. 4443

35 Schneeweiss S. Sensitivity analysis and external adjustment for unmeasured confounders in epidemiologic database studies of therapeutics. Pharmacoepidemiol Drug Saf 2006;15:291-303. doi:10.1002/pds.1200

Web appendix: Supplementary online content 